\title{
VOLVER A PENSAR LA PEDAGOGÍA DE LA EDUCACIÓN FÍSICA ESCOLAR: EXPERIENCIA, SABER Y PRÁCTICA PEDAGÓGICA
}

\author{
Alejandra Mariel Ruiz \\ Universidad de Barcelona, Barcelona, España
}

\begin{abstract}
Resumen
Este texto es una invitación a reflexionar sobre la pedagogía de la educación física escolar, a partir de una mirada nueva sobre viejos temas. Experiencia, saber y práctica pedagógica son los términos que nos guían para adentrarnos en el análisis de la enseñanza de los saberes corporales y motrices de la educación física, con el fin de recuperar el carácter reflexivo, subjetivo y de transformación que todo proceso de aprendizaje conlleva. Para ello, se presenta la experiencia como la productora de un saber que supera y desborda el saber de las disciplinas escolares, lo que supone que tanto educadores como estudiantes transiten nuevas experiencias pedagógicas, comenzando por desandar el propio camino transitado como niños, adolescentes, jóvenes y adultos en un sistema escolar regido por los sentidos de una modernidad hoy ausente.

Palabras clave: Experiencia - Saber - Práctica Pedagógica - Educación Física Escolar
\end{abstract}

\section{"Experiencia" y "saber" en la producción de sentido}

La experiencia es lo que nos pasa, o lo que nos acontece, o lo que nos llega. No lo que pasa, o lo que acontece, o lo que llega, sino lo que nos pasa, o nos acontece, o nos llega [...] la experiencia, la posibilidad de que algo nos pase, o nos acontezca, o nos llegue, requiere un gesto de interrupción: [...] requiere pararse a pensar, pararse a mirar, pararse a escuchar, pensar más despacio, mirar más despacio y escuchar más despacio, pararse a sentir, sentir más despacio, demorarse en los detalles, suspender la opinión, suspender el juicio, suspender la voluntad, suspender el automatismo de la acción, cultivar la atención y la delicadeza, abrir los ojos y los oídos, charlar sobre lo que nos 
pasa, aprender la lentitud, escuchar a los demás, cultivar el arte del encuentro, callar mucho, tener paciencia, darse tiempo y espacio. (LARROSA, 2003: 168-174).

$\mathrm{L}$

a intención de este texto es motivar una reflexión sobre el saber y la experiencia en relación con los procesos de enseñanza y aprendizaje de saberes corporales y motrices que la educación física escolar propicia. Dicha reflexión supone rastrear indagaciones de filósofos y pedagogos que vienen desarrollando interesantes estudios acerca de la comprensión de los procesos educativos y humanos.

Al reflexionar sobre la pedagogía de la educación física, he caído en la cuenta de que la experiencia y el saber se encuentran en una relación muy particular, que resulta esencial para comprender la realidad que les toca vivir a niños y niñas (y cuando digo 'les toca vivir' me refiero a que no son ellos los que deciden cuándo, dónde y de qué modo nacer, como tampoco en qué condiciones materiales y sociales crecer, aunque sí pueden hacer elecciones dentro de dichas condiciones materiales y sociales, no sin ciertos costos personales). La relación a la que me refiero no es la que se idealiza cuando se piensa en la apropiación de conocimientos circunscriptos a los saberes encerrados en las disciplinas escolares ${ }^{1}$. Esos saberes son formas organizadas de comunicar y de especificar algunas verdades que representan ciertas formas de conocer el mundo dentro de la lógica de la cultura occidental. Para establecer una relación más adecuada y abarcadora entre saber y experiencia, primero hay que definir ambos conceptos.

Me pareció oportuno tomar las reflexiones al respecto de autores como Calmels (2001), Contreras (2008) y Larrosa (2003, 2007). Los tres, a su manera, conciben la experiencia como una forma compleja de conocimiento, a partir de una relación dialéctica entre el mundo y el sujeto que lo recibe, lo crea y lo recrea. Dicha relación dialéctica supone formas especializadas y diversas de construir saberes y sujetos, pero la experiencia además constituye, como sostiene Larrosa (2007, p. 7), "una forma de hablar desde la vida", así como la docencia tiene algo que ver con las "ganas de vivir" y por ende con las "ganas de hacer vivir". En ese sentido, la experiencia tiene que ver con lo

1-Las complejas razones por las que los conocimientos aparecen socialmente organizados en disciplinas incumben simultáneamente a la historia de las ciencias, a la historia de la educación y a la sociología del conocimiento. Cf. Cullen (2008).

Pensar a Prática, Goiânia, v. 13, n. 1, p. 1-13, jan./abr. 2010 
que nos pasa, es decir, con aquello que nos sobrepasa, con lo que nos es exterior, ajeno, nuevo. La experiencia siempre es experiencia de algo:

la experiencia exige un acontecimiento, exige el pasar de alguna cosa que no soy yo [...] y eso de que la experiencia es siempre experiencia de algo que no soy yo, significa también que es algo que no depende de mí, es decir que no es una proyección de mí mismo, no es una proyección de lo que yo ya sé, ni de lo que yo pienso, ni de lo que yo ya siento, etc. [...] no será nunca sujeto de la experiencia ese tipo de personas que encuentren lo que encuentren siempre se encuentran consigo mismas, es decir con una proyección de lo que ya saben, de lo que ya piensan, de lo que ya creen, de lo que ya sienten, de lo que de alguna manera anticipan. (LARROSA, 2007, p. 7)

Tal es la primera condición de la experiencia: un momento de exterioridad, alteridad y alienación. Cada una de estas cualidades hace con que la experiencia nos resulte algo tanto ajeno y extranjero (exterioridad) como diferente del propio acontecer (alteridad), y también como algo de lo que uno jamás se puede terminar de apropiar (alienación). La segunda condición de la experiencia es un proceso reflexivo y subjetivo de transformación: reflexivo, porque toda experiencia somete a quien la vive a un salir de sí, enfrentarse a algo ajeno y novedoso, para luego regresar al lugar del yo; subjetivo, porque la experiencia afecta al sujeto de alguna manera (de lo contrario, no se trataría de una experiencia, sino de una repetición). Por ende, la experiencia supone un sujeto abierto, vulnerable y sensible, que elabora en relación con las experiencias de otros su propio saber, singular y concreto. Ese sujeto abierto y sensible es además capaz de formarse y transformarse.

Toda experiencia es ante todo corpórea, ya que no hay vida sin cuerpo, y tampoco hay vida sin 'otro' (el cuerpo materno todavía es la condición previa de todo cuerpo, pese a los avances tecnológicos). Ese cuerpo ajeno es la primera experiencia de vida, el lugar donde se construye lo que nos pasa, y donde se va creando un 'saber' significado subjetivamente y difícil de generalizar, cuyo sentido es una pregunta tan abierta como la pregunta por el valor de lo vivido. La experiencia del cuerpo como primer contacto con el mundo permite 
adquirir saberes que no requieren desligar lo vivido de la actividad del pensamiento, pero que tampoco lo reducen a repetirlo, ni a atribuirle significados preestablecidos. La experiencia del cuerpo busca para este pensar, como para el propio vivir, sus propias respuestas.

Calmels (2001) sostiene que el saber corporal supone una forma de conocimiento de uno mismo que parte de la experiencia propia de vivir en el mundo, un saber inacabado e incompleto que se va completando a partir de prácticas. El saber corporal es ante todo práctico: saber es saber hacer, aunque en nuestra cultura siempre ha sido más importante el conocimiento, y más aún el discurso conceptual.

Para Calmels (2001, p. 23), el saber se caracteriza por ser "el producto de una experiencia con el objeto". En particular, el saber sobre el cuerpo, es decir, la representación que construimos sobre nuestro cuerpo se basa en las experiencias que vivimos. Ese saber se traduce en la elección de acciones y posturas que constituyen experiencias hechas carne. Al caminar, por ejemplo, elegimos una forma de apoyar el peso del cuerpo, seleccionamos un ritmo y adoptamos una actitud que corresponde a una forma de posicionarnos frente a los demás. Calmels ve en este posicionamiento una decisión particular (no abstracta, ni ocasional) que deja traslucir una manera única de establecer con otros una relación que, a su vez, es un entrecruzamiento de relaciones construidas sobre experiencias vividas:

[...] la postura está relacionada con la huella que ha dejado otro (p.ej. la madre); en presencia de un nuevo otro lo que uno hace es acomodar la percepción que habilita los ojos a la mirada, el oído a la escucha y la mano al contacto. Por ende la experiencia no se construye sólo en actos de pensamiento sino desde la acción, desde el hacer con el cuerpo y desde el cuerpo. En esa experiencia motriz el niño y la niña expresan su ser y se entiende como un diálogo con el entorno la manifestación que establece el ser. Por tanto aquí la experiencia se transforma en una dialéctica de la corporalidad con el mundo. (CALMELS, 2001, p. 29)

La corporalidad nos remite a esa unidad perceptiva que establece nexos con la realidad, y que por lo tanto está vinculada al otro en la interacción descrita por Merleau Ponty (1985 [1945]). Desde este punto de vista, el saber se construye a partir de la experiencia de un mundo percibido por un sujeto que lo hace suyo. 
Es desde el cuerpo, interpretado como un locus desde el cual se experimenta y se construye un saber en contacto con el mundo, que se sobrepasa el conocimiento disciplinar elaborado en campos teóricos desvinculados de las posibilidades de aprendizaje desde lo humano. El saber corporal desborda ese conocimiento científico: es una construcción que incluye lo sensorial, lo social, lo temporal y lo espacial, en una suerte de elaboración compleja e integrada a partir de su intelectualidad. De ese modo, saber y experiencia se entrelazan en una relación de mutua reciprocidad y empatía que no puede quedar al margen de ninguna práctica pedagógica y mucho menos de la práctica pedagógica de la educación física.

\section{Del saber pedagógico en la educación física escolar}

En el oficio docente el maestro no realiza su tarea con los apuntes de didáctica bajo el brazo; ni siquiera 'sabiéndose' los temas de la didáctica; que lo que sea que tenga como saber pedagógico, es lo que lleva puesto, incorporado, en su sentido literal, esto es, metido en el cuerpo, hecho cuerpo [...]. (CONTRERAS, 2008, p. 6)

Teniendo en cuenta los conceptos expuestos anteriormente, sostengo que la pedagogía de la educación física debería tomar un nuevo camino, marcando una distancia respecto de las conceptualizaciones tradicionales de las pedagogías ${ }^{2}$ de la educación física que, como intervenciones sistemáticas e intencionales de prácticas corporales y motrices con propósito educativo, hasta el presente han introducido en la escuela una concepción dualista del sujeto y una concepción positivista del movimiento (ambas subsidiarias de otros campos de prácticas motrices, deportivas y gimnásticas ${ }^{3}$ ).

La pedagogía de la educación física, dominada por un pensamiento positivista y simplificador, se halla hoy frente a severas limitaciones para entender la singularidad de sus destinatarios, así como la heterogeneidad y la incertidumbre que se genera en las propias prácticas pedagógicas frente a diversos contextos.

2-Utilizo el término pedagogías, en plural, ya que ha habido diversos modos de intervención intencionada y sistematizada de educar corporal y motrizmente al hombre a lo largo de la historia de la Educación Física. 
Si bien el surgimiento de un pensamiento complejo ha hecho posible repensar a la educación física como una pedagogía de la corporeidad y la motricidad, es decir, como la intervención intencional y sistematizada sobre la constitución corporal y motriz de sujetos íntegros y situados, abandonando la idea del sujeto entendido como máquina biológica o como cuerpo entrenable, es un saber que todavía está por construirse. Esto plantea a docentes y educadores el desafío de la deconstrucción de las propias experiencias, para reconstruir una pedagogía de la educación física basada en una experiencia pedagógica que tenga en cuenta otras vinculaciones posibles entre los saberes de los sujetos y los saberes de las disciplinas. Ello se traduce, a su vez, en la posibilidad de establecer otras relaciones entre la teoría y la práctica, ante la presencia de sujetos con distintas experiencias de aprendizaje.

El saber pedagógico que sucede en el encuentro de experiencias no olvida su vinculación con la vivencia pedagógica. Es un saber que sucede sobre el acontecer, sobre las experiencias que se transitan, y que conduce al auténtico conocimiento pedagógico, aquel que se traduce en pensamiento actuante. Se trata de

un saber que insinúa, que ofrece la experiencia en su apertura a ser pensada y a actuar como inspiración, sin sustituir el movimiento que tiene que hacer cada cual para que conecte con su propia vivencia, para que inspire su propio pensar y hacer [...] es un saber que, a veces, lo que tiene que hacer es callar, crear el vacío en el que el otro pueda hacer emerger su propia verdad [...] una verdad que está tan sólo naciendo; que sólo podrá ser si llega a ser experiencia propia. (CONTRERAS, 2008, p. 4).

Al respecto, María Zambrano (2000, p. 73-74) agrega: "Vivimos una época en la que estamos llenos de ciencia y de técnicas, pero pobres, inmensamente pobres, de todas las formas activas, actuantes del conocimiento". Zambrano se refiere a esa forma de conocer que es necesaria para vivir, o mejor dicho, para emprender la vida con una in-

3-“En los primeros años del s. XX se sostuvo que el ejercicio corporal ofrecía una ocasión de reposo a los niños dedicados a los 'trabajos del espíritu y la mente' aclarando que se elegían para ello ejercicios fáciles que no obligasen a las facultades intelectuales a entrar en juego" (VÁZQUEZ, 1996, p. 45). 
tuición, una visión, una guía: se trata del saber de la experiencia, que orienta nuestra vida porque no se despega de ella. Es un tipo de saber y una necesidad de saber, dice Zambrano (ídem, p. 83), que no es ciencia ni aspira a serlo, que se resiste "a ascender al cielo de la objetividad", porque la vida necesita de su verdad. Es un saber apegado al vivir y a la experiencia, porque se conecta con la vivencia propia de quien lo comunica y tiene que tener la posibilidad de conectarse también con la vivencia propia de quien lo escucha. Es un saber que no queda completamente expresado como un mero conjunto de proposiciones; que es persuasivo, pero que no lo dice todo, ni puede hacerlo; que afirma que hay cosas que no pueden decirse, y que sólo pueden insinuarse, o a veces callarse: "Para que el otro sienta nacer dentro de sí lo que necesita y sea más suyo; para que lo sepa por experiencia también, para que el que escucha encuentre dentro de sí, en status nascens, la verdad que necesita"4.

Planteada en este sentido, la pedagogía recupera su tarea de planificar una intervención intencional para incidir en la formación compleja de los sujetos y en la apropiación de los bienes culturales específicos, desde prácticas docentes que consideren la complejidad y la totalidad humana, hasta posibles formas de comprender y transformar la realidad individual y social, incorporando, al hacerlo, otros componentes que se rescaten de la realidad social y cultural como elementos que sitúen al aprendizaje en contexto.

\section{A modo de reflexión final}

Es hora de comenzar a pensar juntos en una pedagogía de la educación física más sensible. En base a la lógica desarrollada en este trabajo, es posible ofrecer una aproximación: la pedagogía, en su dimensión práctica, pone en juego tanto aquello que despliega el educador (quien, como sostiene Meirieu (1998), es el adulto encargado de llevar adelante la función de 'hacer' al infante), como aquello que despliegan los diversos 'yo' experienciales (quienes, respetando su naturaleza situa$\mathrm{da}$, ponen en entredicho constantemente lo que el 'yo' impuesto pretende que sean).

4-Max Van Manen insiste en que "[la] pedagogía 'consiste' en este constante cuestionamiento; es este dudar siempre. Nos preguntamos: ihe hecho lo correcto?" (VAN MANEN, 2003, p. 162; cf. también VAN MANEN, 1998, 2004). 
Esto hace que la pedagogía, como práctica y espacio de relación entre niños y niñas y entre alumnos y docentes, se vuelva a investigar desde el punto de vista de la corporeidad docente, considerando a la docencia como un campo que se ha consolidado desde ciertos simbólicos femeninos y masculinos dominantes. Los educadores deberían repensar la diferencia que implica la construcción de una corporeidad que ha sido simbólicamente construida a partir de la mirada de lo masculino, así como también deberían estar abiertos a incorporar las múltiples diferencias que nos constituyen subjetivamente, tal como plantea Rosi Braidotti (2000). De lo contrario, quedaremos atrapados en otro relato universal simplificador, meramente actualizado para un nuevo estado del mundo.

La práctica desarrollada en campos escolares, los cuales, como sostiene Cullen (2008, p. 53), son el "lugar de producción y circulación de conocimientos socialmente válidos y significativos", supone enfrentar el doble desafío de, por un lado, transmitir contenidos (aquello que se enseña y que se espera sea aprendido), y por otro lado, crear contenidos que habiliten a los sujetos para 'ser'.

Con esta doble intención, no se puede ir por caminos separados ni atender a un mismo sujeto como si fueran dos diferentes. El desafío debe pensarse como parte de una misma tarea pedagógica, encargada de la transmisión de conocimientos legitimados públicamente, pero a la luz de nuevas reconceptualizaciones que tengan en cuenta algo que por momentos la historia de la pedagogía y de la escuela ha olvidado: los 'saberes previos' al ingreso a la escuela, es decir, los saberes culturalmente construidos por los sujetos individuales y particulares. La suerte de ese reconocimiento puede de alguna manera evitar la censura o, lo que es peor, la autocensura que surge de la identificación del yo con los grandes relatos oficiales de la cultura, que pretenden ayudar al individuo a convivir con los demás.

Los grandes relatos han funcionado a modo de cauce para los cuerpos y sus diferencias de todo tipo (sexuales, étnicas, de género, culturales, etc.), colocándolos en los carriles correspondientes, posibles y esperados, aún cuando ello fuera en contra de la voluntad o del propio deseo. Como grandes relatos, la pedagogía, y en particular la pedagogía de lo corporal, han inscripto a lo largo de la historia los modos y las formas de encauzar esos deseos, incluso para aquellos que no son pasibles de ser contenidos.

En ese sentido, Cullen (2008, p. 39-40) ha señalado las dificultades 
y las consecuencias del modelo enciclopedista de organización de contenidos:

Dicho modelo ha contribuido a la construcción de un sujeto social del conocimiento escindido de sí mismo, desfasado del sujeto cultural que lo sostiene; sostuvo como criterio de control social para la circulación del conocimiento el 'despojo' de saberes previos (y con ellos de contextos socio-económicos diferentes) y la consigna de la neutralidad y de la normalidad de los saberes enseñables (y con ello la exclusión de la historicidad, el conflicto y la crítica); finalmente se ilusionó pensando que con la adaptación a la sociedad unidimensional y a sus valores hegemónicos se lograrían los fines sociales de la educación, que una retórica repetida y ampulosa formulaba, y sigue formulando, como 'formación integral del hombre y del buen ciudadano'.

Este cuestionamiento del modelo hegemónico exige repensar además los cimientos de toda pedagogía y, por sobre todo, el pensamiento de los docentes acerca de los niños y las niñas, la escuela, el conocimiento y el saber, a la luz de los nuevos escenarios sociales. Evidentemente, si la pedagogía (y la educación) aceptan reconocer un sujeto corporalmente situado, fragmentado, disperso, móvil y con saberes válidos, deberán recrear su concepción de las relaciones del adulto con la infancia y con el conocimiento. Es posible que en el proceso las prácticas pedagógicas se conviertan en recorridos respetuosos, donde niños y niñas puedan elaborar sus propias prácticas con sentido, ya que aprenderán distintos sentidos desde diferentes historias, y desde diferentes estrategias de apropiación y contrastación social de los saberes y conocimientos enseñados (CULLEN, 2008).

En definitiva, toda práctica pedagógica, más allá de estar elaborada sobre la base de un tipo de relato pedagógico, se sostiene en base al encuentro de diferencias encarnadas en los sujetos que permiten el reconocimiento del 'otro'. Cuando los relatos no ceden espacios para la construcción subjetiva, lo 'otro' (lo que está afuera, producto del discurso que habilita o inhabilita) se vive como aquello que difiere y merece una mediación temporalizadora (consciente o inconsciente), y que tiene por finalidad, la mayoría de las veces, suspender el propio deseo creando uno nuevo, lo cual anula el efecto (DERRIDA, 1989). 
Una pedagogía interesada en el reconocimiento y la valoración de las diferencias constituyentes de todo 'otro' tampoco puede huir del desafío de enfrentar la constitución de corporeidades 'otras', fluidas y móviles, marcadas por actos de identificación a lo largo de sus trayectorias de vida. Debe indagar en los siguientes interrogantes: ¿Cómo romper con binarismos y pensar la sexualidad, los géneros y los cuerpos de una forma plural, múltiple y cambiante? ¿Cómo conducir la mirada hacia prácticas pedagógicas que -en palabras de López Louro (2001, p. 12), no se limiten a 'soportar' la diferencia, sino que la produzcan como sinónimo de una presencia activa en la escuela y en la vida social de los sujetos.

La pedagogía, entonces, debe abrir espacios de indeterminación de la corporeidad, es decir, espacios desconocidos para acceder, a los cuales se necesita un reconocimiento de las diferencias que nos conforman como sujetos y que muchas veces están determinadas por condiciones que van más allá de las clases sociales, la etnia, el género, la sexualidad y la nacionalidad 5 .

Rethinking physical education at school: experience, knowledge and pedagogical practice

\begin{abstract}
The present paper is an invitation to think about the pedagogy of physical education at school, taking a new look at old themes. Experience, knowledge and pedagogical practice are some of the terms that will guide us as we undertake the analysis of different ways of teaching bodily knowledge and developing motor skills in the realm of physical education, in order to restore the thoughtful, subjective and transformative character that every learning process should entail. For this purpose, the paper introduces the concept of experience as the source of a distinctive kind of knowledge that surpasses and overflows the contents of traditional school subjects. This reassessment of children's insightfulness concerning their bodies demands that both educators and students take part in new pedagogical experiences. To begin with, it is necessary that they trace back their steps on the path they followed as children, teenagers and adults, because they were subjected to an educational system ruled by a certain set of meanings, which were underpinned by modern values that no longer exist.
\end{abstract}

Keywords: Experience - Knowledge - Pedagogical Practice - Physical Education

Repensar a pedagogía da educação física escolar: experiência, saber e prática pedagógica

5-Dussell sostiene que estos son sólo algunos de los componentes sociales que delimitarían el margen 


\section{Resumo}

Este texto é um convite à reflexão sobre o ensino da educação física na escola, a partir de um novo olhar sobre velhos temas. Experiência, saber e prática pedagógica são os termos que nos orientam para adentrarmos na análise do ensino dos saberes corporais e motrizes da educação física, com o objetivo de recuperar o caráter reflexivo, subjetivo e de transformação que todo processo de aprendizagem acarreta. Para tanto, se apresenta a experiencia como a produtora de um saber que supera e ultrapassa o saber das disciplinas escolares, e isso supõe que tanto educadores quanto aprendizes veiculem novas experiencias pedagógicas, começando por desvendar o próprio caminho transitado enquanto crianças, adolescentes, jovens e adultos em um sistema escolar regido pelos sentidos de uma modernidade atualmente ausente.

Palavras chave: Experiência - Saber - Prática Pedagógica - Educação Física Escolar

\section{Referencias}

BRAIDOTTI, R. Sujetos nómades. Buenos Aires: Paidós, 2000.

CALMELS, D. Cuerpo y saber. 3. ed. Buenos Aires: Novedades Educativas, 2001.

CONTRERAS, D. Acerca de la experiencia y experiencia, escritura y deliberación: explorando caminos de libertad en la formación didáctica del profesorado. In: Congreso Internacional de Formación Docente, 3. Buenos Aires, 2008.

CULLEN, C. Críticas a las razones de educar: temas de filosofía de la educación. Buenos Aires: Paidós, 2008.

DERRIDA, J. La différance. In: Márgenes de la Filosofía. Madrid: Cátedra, 1989. p. 39-62.

DUSSELL, E. Deconstrucción y latinoamericanismo. Red Maestro de Maestros, Portal Claudia González Castro. Disponible en $<$ http://www.rmm.cl> Acceso en: 27 ene. 2007.

LARROSA, J. Trabajar en la educación tiene que ver con la pasión por el encuentro, un compromiso vital. In: Encuentro Nacional "Formar en Futuro Presente", 7. Barcelona, 20 sept. 2007. [Conferência]

LARROSA, J. Entre las lenguas: lenguaje y educación después de Babel. Barcelona: Alertes, 2003. 
LÓPEZ LOURO, G. Teoría Queer: una política pos-identitaria para la educación. In: Cuadernos de Pedagogía Rosario, Rosario: Laborde Editor/Centro de Estudios en Pedagogía Crítica, n. 9, 2001.

MEIRIEU, P. Frankenstein educador. Barcelona: Alertes, 1998.

MERLEAU-PONTY, M. Fenomenología de la percepción. Barcelona: Planeta Agostini, 1985 [1945].

VAN MANEN, M. EI tacto en la enseñanza: el significado de la sensibilidad pedagógica. Barcelona: Paidós, 1998.

. El tono de la enseñanza: el lenguaje de la pedagogía. Barcelona: Paidós, 2004.

. Investigación educativa y experiencia vivida. Barcelona: Idea Educación, 2003.

VÁZQUEZ, B. La Educación Física en la educación básica. Madrid: Gymnos, 1996.

ZAMBRANO, M. Hacia un saber sobre el alma. Madrid: Alianza, 2000.

Recebido em: 20/10/2009

Revisado em: 24/11/2009

Aprovado em: 03/03/2010

Endereço para correspondência

rumar98@yahoo.com

Alejandra Mariel Ruiz

Universidad Nacional de Lujan

Ruta 5 y Avenida Constitución - (6700) Luján

Buenos Aires, Argentina 